\title{
Development and validation of risk score for predicting spontaneous rupture of hepatocellular carcinoma
}

\author{
Feng Ye*, Di Ma*, Xiao-Yong Gong, Yu-Chen Yang, Yong-Jun Chen \\ Division of Hepatobiliary Surgery, Department of General Surgery, Ruijin Hospital, Shanghai Jiaotong University School of \\ Medicine, Shanghai, China
}

Purpose: Spontaneous rupture is a potentially serious complication of liver cancer. A risk score was developed and validated for predicting spontaneous rupture based on a retrospective study.

Methods: Multiple logistic regression analysis was used to study the relationship between clinical variables and spontaneous rupture. The independent rupture predictors were converted into a score based on the odds ratio. Predicted attributes of the developed scores were then verified using a dataset in 2019.

Results: The incidence of spontaneous rupture was $5.5 \%$ from 2002 to 2019 . A 10 -point score l $\alpha$-FP of $\geq 400 \mu \mathrm{g} / \mathrm{L}, 1$; protrusion from liver surface, 2; ascites, 3; tumor size of $>5 \mathrm{~cm}, 4)$ was derived for prediction of rupture and area under the receiver-operating characteristic curve was 0.9 (95\% confidence interval, 0.87-0.92). When applying a cutoff value of 5 points or more, the specificity was 0.87 and the sensitivity was 0.84 . A validation cohort consisting of 202 hepatocellular carcinoma patients reproduces the predictive, identification, and calibration characteristics. The observed rate of spontaneous rupture according to risk stratification of the score was $0.6 \%$ for those with a score of $0-4,21.6 \%$ for a score of $5-7$, and $36.4 \%$ for a score of $8-10$ in the validation cohort.

Conclusion: Here, based on routine clinical data, we determine the factors that affect prognosis and propose an effective tool for predicting spontaneous rupture, which may be useful in guiding priority treatment of high-risk patients or clinical routine preventive treatment.

[Ann Surg Treat Res 2020;99(5):268-274]

Key Words: Forecasting, Liver cancer, Patient recruitment, Rupture, Scoring methods

\section{INTRODUCTION}

Spontaneous liver rupture is a potentially serious complication of liver cancer and occurs in about 3\%-26\% of hepatocellular carcinoma (HCC) patients [1]. Mortality from spontaneously ruptured $\mathrm{HCC}(\mathrm{rHCC})$ remains high in the acute phase, ranging from $25 \%$ to $75 \%$, and the median survival is short, about 7-21 weeks, making it the third common cause of death of liver cancer excepting cancer progression and liver failure [2,3]. Either nonsurgical or surgical treatment aiming at long-term survival is often considered as futile in treating rHCC [4]. Furthermore, according to the liver allocation policy for liver transplantation for HCC, $\mathrm{rHCC}$ is one of the contraindications to HCC exception points [5]; to downstage rHCC to fulfill the Milan or University of California San Francisco (UCSF) criteria is not an established strategy and is not always feasible [3]. Thus, when rHCC occurs, most of the early HCC patients lose the opportunity for radical treatment. Therefore, great effort is needed to prevent rHCC. In a society with limited healthcare resources, the identification of liver cancer patients with high
Received April 7, 2020, Revised June 25, 2020, Accepted July 29, 2020

Corresponding Author: Yong-Jun Chen

Division of Hepatobiliary Surgery, Department of General Surgery, Ruijin Hospital, Shanghai Jiaotong University School of Medicine, 5F, Building 6, 197 Second Ruijin Road, Shanghai 200025, China

Tel: +86-21-64370045-666052, Fax: +86-21-64314781

E-mail:yongjunchen@yahoo.com

ORCID: https://orcid.org/0000-0002-6486-2000
*Feng Ye and Di Ma contributed equally to this study as co-first authors. Copyright (c) 2020, the Korean Surgical Society

(C) Annals of Surgical Treatment and Research is an Open Access Journal. All articles are distributed under the terms of the Creative Commons Attribution NonCommercial License (http://creativecommons.org/licenses/by-nc/4.0/) which permits unrestricted non-commercial use, distribution, and reproduction in any medium, provided the original work is properly cited. 
risk of rupture will help to determine priority treatment.

Risk factors, such as cirrhosis and tumor size, have been shown to be associated with rHCC $[6,7]$. However, there is no reliable clinical routine scoring system to predict rHCC. Therefore, using the data from Ruijin Hospital in Shanghai between 2002 and 2018, this study established a score to predict the risk of rupture based on the risk factors in $\mathrm{rHCC}$ and validated its predictive properties in an independent dataset in 2019.

\section{METHODS}

\section{Derivation cohort}

From 2002 to 2018, a total of 50 consecutive patients presenting with $\mathrm{rHCC}$ at a tertiary medical center were included in the analysis. A control arm of 886 consecutive patients with nonruptured HCC was used for comparison at the hospital during the same study period. Clinical variables, laboratory test results, radiologic findings, and pathological findings were retrospectively collected.

HCC was diagnosed by one typical radiologic imaging examination showing characteristic features of HCC, or by histologic confirmation. The study defined spontaneous rupture as an event that occurred in the absence of abdominal trauma or iatrogenic injury to the liver. The diagnosis of rHCC was confirmed by imaging showing features of active peritumoral contrast extravasation or intraoperative findings of rupture through contrast-enhanced MRI or CT.

\section{Validation cohort}

Derived estimates were validated in HCC patients who were treated in 2019 at the same hospital. Data collection methods and definition in this group were the same as these of the derivation group.

\section{Statistical analyses}

A risk score was developed in the derived group. First, we defined variables that may predict $\mathrm{rHCC}$ based on clinical experience and literature. Clinical features included demographics (age and sex), routine hematologic and biochemical investigations (routine blood test, liver and kidney function test, and coagulation function test), $\alpha-\mathrm{FP}$, liver function reserve (Child-Pugh classification), ascites, HBV infection status, Barcelona Clinic Liver Cancer (BCLC) classification, vascular invasion (portal vein, hepatic vein, and bile duct), direct invasion of adjacent organs excluding the bladder, underlying disease (cirrhosis and hypertension), type of treatment, occurrence of previous transcatheter arterial chemoembolization (TACE), pathological findings (tumor grade), while imaging parameters including maximum preoperative tumor diameter, number of tumors (solitary or multiple), formation of portal vein tumor thrombus, tumor protrusion from liver surface (PFLS).

To develop the risk score, variables associated with rHCC were identified with a chi-square test for categorical variables and a Student t-test for continuous variables. Statistically significant risk factors for rHCC analyzed by univariable logistic regression that tended to influence occurrence of rupture (defined as a $\mathrm{P}<0.10$ in univariable regression) were subjected to a stepwise backward logistic analysis to identify potential independent predictors for rupture with a cutoff $\mathrm{P}<0.05$ for retention in the model using maximum likelihood function. Two-way interactions between the independent variables were considered. Variables in the model are used to create the rupture score.

Each factor was assigned points based on the appropriate odds ratios (ORs) from the final logistic regression analysis. In order to make the score closer to an integer and be convenient for doctors, the study takes the approximate integer point for each $O R$. The lowest $O R$ value is $28.05,28.05$ times 0.04 equals 1 , so that all $O R$ values are multiplied by 0.04 to make the $O R$ value rounded to the nearest integer. The final score is the sum of all the points. Because of the range of possible scores $(0-10)$, the number of patients with a specific score differs; therefore, the study divided the risk score into 3 groups (based on the estimated probability of rupture) and reported the risk of rupture in each group. Discrimination abilities of the developed score were assessed by area under the receiver-operating characteristic curve (AUC). In addition, the calibration ability of the predictive scoring system was assessed by HosmerLemeshow test, and Nagelkerke $\mathrm{R}^{2}$ was used as a goodness-of-fit measure.

This study verified the model from the derivation group in the validation group. The prediction ability, discrimination ability, and calibration ability of the scoring system have been verified. The validation data set was used to reproduce the observed attributes according to the prediction model. For statistical analysis accuracy, patients with missing data were excluded from the analysis. Statistical significance was determined at an $\alpha$ level of 0.05 and all tests were 2-tailed. Data analyses were performed using IBM SPSS Statistics for Mac (ver. 26.0, IBM Corp., Armonk, NY, USA).

\section{Ethics statement}

The study was in line with the principles set out in the Declaration of Helsinki. All patients signed informed consent for their data to be used for research purposes after clear and complete explanation and consent was recorded in the patients' medical records. The Institutional Review Board of Ruijin Hospital approved this study (No. 2018209). 


\section{RESULTS}

\section{Demographics and clinical characteristics}

Clinical and demographic factors of $50 \mathrm{rHCC}$ patients and 886 consecutive HCC patients without rupture in the derivation cohort were presented in Table 1. There were no significant differences in the statistics concerning age, sex distribution, platelet, total bilirubin, AST, tumor location, tumor grade, vascular invasion, portal vein tumor thrombus, extrahepatic invasion, HBV infection, previous TACE, and BCLC stage (Table 1) between the 2 groups. No significant differences were observed between the groups compared with nonrupture group, more patients in rupture group had $\alpha$-FP of $\geq 400 \mu \mathrm{g} /$ L (39.3\% vs. $10.0 \%, \mathrm{P}=0.002)$, PFLS (76.0\% vs. $8.0 \%, \mathrm{P}<0.001$ ), tumor size of $>5 \mathrm{~cm}(88.0 \%$ vs. $10.0 \%, \mathrm{P}<0.001)$, multifocal lesions ( $15 / 35$ vs. $4 / 46, P=0.005)$, and ascites $(40.0 \%$ vs. $8.0 \%, \mathrm{P}<0.001)$. Patients with rHCC had fewer underlying diseases of cirrhosis $(32.0 \%$ vs. $62.0 \%, \mathrm{P}=0.003)$. It was shown that the following 6 laboratory variables and Child-Pugh classification were associated with rHCC: hemoglobin, albumin, creatinine, ALT, PT, and international normalized ratio (Table 1). However, these variables were not kept in the univariable analysis because these laboratory tests were done on rHCC patients after rupture, which means the laboratory difference is a consequence, rather than the cause of rupture.

\section{Risk factors of rHCC and development of a risk score}

A total of 1,138 HCC patients were admitted to Ruijin Hospital from 2002 to 2019 and 63 patients were diagnosed with rHCC,

Table 1. Demographic and clinical characteristics of patients with and without spontaneous rupture of hepatocellular carcinoma in the derivation cohort

\begin{tabular}{|c|c|c|c|}
\hline Characteristic & Rupture $(n=50)$ & Nonrupture $(\mathrm{n}=886)$ & P-value \\
\hline Age (yr) & $57.2 \pm 16.8$ & $59.5 \pm 11.9$ & 0.347 \\
\hline Sex, male/female & $41 / 9$ & $603 / 283$ & 0.038 \\
\hline \multicolumn{4}{|l|}{ Laboratory data } \\
\hline Hemoglobin $(g / L)$ & $99.1 \pm 25.5$ & $134.59 \pm 17.8$ & $<0.001$ \\
\hline Platelet $\left(10^{9} / \mathrm{L}\right)$ & $174.2 \pm 94.4$ & $149.4 \pm 70.3$ & 0.021 \\
\hline Albumin (g/L) & $31.0 \pm 6.1$ & $38.9 \pm 15.0$ & $<0.001$ \\
\hline Total bilirubin $(\mu \mathrm{mol} / \mathrm{L})$ & $32.8 \pm 50.1$ & $19.4 \pm 17.1$ & 0.077 \\
\hline Creatinine $(\mu \mathrm{mol} / \mathrm{L})$ & $124.1 \pm 137.9$ & $77.2 \pm 81.9$ & 0.034 \\
\hline AST (IU/L) & $377.3 \pm 1,359.9$ & $60.3 \pm 118.4$ & $<0.001$ \\
\hline ALT (IU/L) & $127.6 \pm 232.7$ & $37.1 \pm 35.2$ & 0.011 \\
\hline PT (sec) & $15.5 \pm 3.5$ & $13.1 \pm 2.4$ & $<0.001$ \\
\hline INR & $1.3 \pm 0.3$ & $1.0 \pm 0.1$ & $<0.001$ \\
\hline \multicolumn{4}{|l|}{ Tumor-related factor } \\
\hline$\alpha-\mathrm{FP} \geq 400 \mu \mathrm{g} / \mathrm{L}$ & $11(22.0)$ & $138(15.6)$ & 0.001 \\
\hline Location, $\mathrm{L} / \mathrm{R} / \mathrm{B} / \mathrm{C}^{\mathrm{b})}$ & $25 / 20 / 2 / 3$ & $496 / 356 / 17 / 17$ & 0.171 \\
\hline Protrusion from the liver surface & $38(76.0)$ & $121(13.7)$ & $<0.001$ \\
\hline Tumor size $>5 \mathrm{~cm}$ & $44(88.0)$ & $154(17.4)$ & $<0.001$ \\
\hline Multifocality, solitary/multiple & $35 / 15$ & 798/88 & $<0.001$ \\
\hline \multicolumn{4}{|l|}{ Pathological findings } \\
\hline Tumor grade, I and II/III and IV & $31 / 19$ & $435 / 327$ & 0.266 \\
\hline Vascular invasion & $2(4.0)$ & $52(5.9)$ & 0.581 \\
\hline Portal vein tumor thrombus & $5(10.0)$ & $118(13.3)$ & 0.499 \\
\hline Extrahepatic invasion & $3(6.0)$ & $34(3.8)$ & 0.445 \\
\hline \multicolumn{4}{|l|}{ Underlying disease } \\
\hline HBV & $24(48.0)$ & $659(75.8)$ & 0.839 \\
\hline Liver cirrhosis & $16(32.0)$ & $541(61.1)$ & $<0.001$ \\
\hline Hypertension & $11(27.5)$ & $320(36.1)$ & 0.266 \\
\hline Ascites & $20(40.0)$ & $88(9.9)$ & $<0.001$ \\
\hline Previous TACE & $1(2.0)$ & $107(12.1)$ & 0.065 \\
\hline Child-Pugh, A/B/C & $20 / 28 / 2$ & $793 / 84 / 0$ & $<0.001$ \\
\hline $\mathrm{BCLC}$ stage, $\mathrm{A} / \mathrm{B} / \mathrm{C} / \mathrm{D}$ & $29 / 13 / 7 / 1$ & $593 / 191 / 82 / 20$ & 0.546 \\
\hline
\end{tabular}

Values presented as mean \pm standard deviation or frequency $(\%)$.

INR, international normalized ratio; TACE, transcatheter arterial chemoembolization; BCLC, Barcelona Clinic Liver Cancer.

${ }^{a} \mathrm{P}$-value corresponds to independent t-test or Mann-Whitney U-test (continuous data) and Pearson chi-square test (categorical data). ${ }^{\text {b) }}$ Left lobe/right lobe/bilobar distribution/caudate lobe. 
Table 2. Risk factors of spontaneous rupture of hepatocellular carcinoma

\begin{tabular}{|c|c|c|c|c|c|c|c|}
\hline \multirow{2}{*}{ Variable } & \multicolumn{3}{|c|}{ Univariable analysis } & \multicolumn{3}{|c|}{ Final multivariable analysis } & \multirow{2}{*}{$\begin{array}{l}\text { Risk } \\
\text { score }\end{array}$} \\
\hline & $\beta$ coefficient & P-value & OR $(95 \% \mathrm{Cl})$ & $\beta$ coefficient & P-value & OR $(95 \% \mathrm{Cl})$ & \\
\hline \multicolumn{8}{|l|}{$\alpha-\mathrm{FP}(\mu \mathrm{g} / \mathrm{L})$} \\
\hline$<400$ & 0 (reference) & & 1 & 0 (reference) & & 1 & 0 \\
\hline$\geq 400$ & 1.762 & 0.004 & $5.824(1.762-19.245)$ & 3.334 & 0.030 & $28.053(1.378-571.260)$ & 1 \\
\hline \multicolumn{8}{|l|}{ PFLS } \\
\hline No & 0 (reference) & & 1 & 0 (reference) & & 1 & 0 \\
\hline Protrusion & 3.595 & $<0.001$ & $36.417(10.855-122.173)$ & 4.103 & 0.007 & $60.502(3.124-1,171.867)$ & 2 \\
\hline \multicolumn{8}{|c|}{ Tumor size $(\mathrm{cm})$} \\
\hline$\leq 5$ & 0 (reference) & & 1 & 0 (reference) & & 1 & 0 \\
\hline$>5$ & 4.190 & $<0.001$ & $66.000(18.769-232.088)$ & 4.715 & 0.004 & $111.652(4.502-2,769.065)$ & 4 \\
\hline \multicolumn{8}{|l|}{ Multifocality } \\
\hline Solitary & 0 (reference) & & 1 & 0 (reference) & & 1 & 0 \\
\hline Multiple & 1.595 & 0.008 & $4.929(1.503-16.157)$ & & & & \\
\hline \multicolumn{8}{|l|}{ Liver cirrhosis } \\
\hline No & 0 (reference) & & 1 & 0 (reference) & & 1 & 0 \\
\hline Cirrhosis & -1.243 & 0.003 & $0.288(0.127-0.658)$ & & & & \\
\hline \multicolumn{8}{|l|}{ Ascites } \\
\hline No & 0 (reference) & & 1 & 0 (reference) & & 1 & 0 \\
\hline Ascites & 2.037 & 0.001 & $7.667(2.384-24.650)$ & 4.544 & 0.035 & $94.074(1.363-6,493.266)$ & 3 \\
\hline Constant & & & & -6.864 & 0.002 & & \\
\hline
\end{tabular}

OR, odds ratio; $\mathrm{Cl}$, confidence interval; PFLS, protrusion from the liver surface.

$\mathrm{P}$-value corresponds to logistic regression analysis. Only the variables with significant influence in the regression model $(\mathrm{P}<0.1)$ were considered in the final models. Variables were eliminated using backward elimination procedure. The score for each factor is the OR multiplied by 0.036 and rounded to the closest integer.

Table 3. Evaluation of discrimination and calibration abilities of the risk score

\begin{tabular}{lcc}
\hline \multicolumn{1}{c}{ Variable } & Derivation cohort & Validation cohort \\
\hline Discrimination & & $0.864(0.794-0.934)$ \\
AUC $(95 \% \mathrm{Cl})$ & $0.894(0.866-0.922)$ & 0.121 \\
Calibration & 0.106 & 0.318 \\
Hosmer-Lemeshow test (P-value) & 0.312 & \\
Nagelkerke $\mathrm{R}^{2}$ & & \\
\hline
\end{tabular}

AUC, area under the receiver-operating characteristic curve; $\mathrm{Cl}$, confidence interval.

with a resulting overall spontaneous rupture rate of $5.5 \%$. A multivariable logistic regression model confirmed that the following factors were significantly related to spontaneous rupture and reached the previously defined $\mathrm{P}<0.05$ indicating clinical significance (Table 2): $\alpha$-FP of $\geq 400 \mu \mathrm{g} / \mathrm{L}$, PFLS, ascites, and tumor size of $>5 \mathrm{~cm}$. According to the ORs obtained from the regression analysis, the scoring system was established by integrating the independent risk factors of rHCC. Points assigned for each factor (APAS: $\alpha$-FP of $\geq 400 \mu \mathrm{g} / \mathrm{L}, 1$; PFLS, 2 ; ascites, 3; tumor size of $>5 \mathrm{~cm}, 4$ ) were present in Table 2; thus, a 10-point score was derived.

\section{Validation}

In the derivation cohort, Table 3 evaluated discrimination and calibration abilities of the risk score with an AUC of 0.89 (95\% confidence interval [CI], 0.87-0.92). For validation, 202 HCC patients admitted consecutively to Ruijin Hospital in 2019 were considered, with AUC was 0.86 (95\% CI, 0.79-0.93) and showed that APAS score predicted rHCC well (Table 3). The appropriate cutoff score for rupture prediction was 5 with a Youden index of 0.708 , sensitivity of $84.0 \%$, and specificity of $86.8 \%$. There was no significant difference between observed and predicted probability, which was confirmed by a HosmerLemeshow test $(P=0.106)$. In the validation cohort, based on an estimated probability of rHCC of $<5.40 \%, 8.54 \%-19.99 \%$, and $>29.01 \%$, individuals with rupture scores of $\geq 8,5-7$, and $\leq 4$ were stratified into high-, intermediate-, and low-risk groups, respectively (Table 4). 
Table 4. Validation: observed rate and estimated probability of spontaneous rupture by risk level when applied to the validation cohort

\begin{tabular}{lccc}
\hline \multicolumn{1}{c}{ Variable } & \multicolumn{2}{c}{ Risk group } & High \\
& Low & Intermediate & $8-10$ \\
Score range & $0-4$ & $5-7$ & $29.01-52.22$ \\
Score-based estimated probability range (\%) & $0.79-5.40$ & $8.54-19.99$ & 36.4 \\
Observed rate of rHCC (\%) & 0.6 & 21.6 & 4 \\
No. of patients with rHCC & 1 & 8 & $11(5.1)$ \\
Patients in risk range (\% of all patients) & $157(73.0)$ & $37(17.2)$ & \\
\hline
\end{tabular}

rHCC, spontaneous rupture of hepatocellur carcinoma.

\section{DISCUSSION}

The aim of present study was to develop a clinical tool for the prediction of rHCC. The performance of a score was evaluated by its discriminatory power [8]. Furthermore, it is necessary to verify the validity of the score by applying it to patients independent from the derivation group [9]. Discrimination was measured by AUC [10]. It is generally defined as useful if AUC is $>0.7$ and is defined as excellent if AUC is between 0.8-0.9 considering the diagnostic accuracy [11]. It could be regarded as excellent to predict a spontaneous rupture of multifactorial origin with the AUC for APAS score at 0.89 and 0.86 for the derivation and validation cohort, respectively. The points assigned for the score value were based on OR values of the regression analysis. As far as we know, this is the first study providing a score for spontaneous hepatic rupture prediction derived from routine variables that were validated within an independent cohort with appropriate discrimination power.

A number of factors that are independently related to $\mathrm{rHCC}$ have been identified by previous studies, further strengthening the confidence with which APAS score might be applied in clinical practice. Consistent associations were shown for PFLS and tumor size of $>5 \mathrm{~cm}[2,12]$. Tumor size has been recognized as an important risk factor for spontaneous rupture, although this may be due to other related variables, such as vascular invasion and tumor grade, rather than the absolute maximum tumor size [12,13]. HCC patients with PFLS had an increased risk of rHCC, as compared with other tumor locations [7,14]. These indicated that normal parenchyma surrounding liver cancer can protect tumors from rupture; thus, protruded tumors without surrounding parenchyma may easily rupture due to compression or friction with adjacent organs $[2,15]$. Interestingly, ascites remained predictive for $\mathrm{rHCC}$. In a nationwide study from Japan and a retrospective study from Thailand, ascites has been shown to be an independent risk factor of rHCC. However, the explanations of why HCC with ascites is more likely to rupture are still questionable $[2,6,16]$. Higher $\alpha-\mathrm{FP}$ had contributed to the risk of $\mathrm{rHCC}$ in this study. This result does support similar findings regarding $\alpha$-FP from another retrospective study on rHCC [6]. However, increasing serum concentrations of $\alpha$-FP may also be correlated with larger tumor size [17]. Overall, mechanisms behind these associations require further investigation, and potential issues related to venous congestion and vascular injury may play a part $[2,18]$.

APAS score mainly predicts the probability of spontaneous rupture, but it is not clear whether individuals with HCC will rupture spontaneously in the future. Therefore, the main value of the scoring system lies in screening high-risk patients and giving preventive measures to high-risk patients. In developing countries with limited medical resources, individuals with liver cancer always develop in the presence of advanced chronic hepatic disease related to HBV. The shortage of hospital beds and grafts available for treatment, however, precludes prompt admission for HCC patients. In an effort to prioritize hospital admission, APAS score helps to identify high-risk patients with a potential value to being a triage marker for HCC. When the predicted risk reaches a certain cutoff level, priority treatment can be considered. Of the risk factors included in APAS score, only ascites are within the reach of intervention. Efforts should be made to triage HCC patients with such factors as PFLS or tumor size of $>5 \mathrm{~cm}$ to more aggressive treatment to reduce the risk of rHCC. HCC patients with high APAS scores should avoid strenuous sports, emotional disturbance, and activities that may lead to liver trauma [19]. There is not a lot of evidence for the effect of preventive measures and priority treatment on the reduction of risk of $\mathrm{rHCC}$ considering the limitations of research methods; it is unethical to design prospective studies with $\mathrm{rHCC}$ due to the fact that it would be unethical not to prevent individuals with higher spontaneous rupture risk, as rHCC is an extremely serious complication. Benefits would outweigh the side effects if the initiation of prophylactic measures and triage could be applied in those with high APAS scores.

A major limitation of our study is that the small sample size of the validation cohort, a larger study population is needed to confirm that APAS score could be used for rupture prediction in HCC cases. Some other limitations of APAS score also need to be discussed. First, as a large proportion of the patients in 
our study population are HCC cases related to hepatitis B, it is necessary to carry out external validation research on the general application of APAS score in Western populations in the future [1,4]. Second, the diagnosis of PFLS was reviewed independently by 2 radiologists and no standardized criteria were applied. There is no specific definition that a location is classified as PFLS even in imaging-based scoring systems such as Liver Imaging-Reporting and Data System (LI-RADS) [20]. However, protrusion has been confirmed to be an independent risk variable for the spontaneous rupture of HCC in previous studies [7].

In conclusion, the present study developed a validated APAS score for spontaneous HCC rupture prediction using a respective database, and the findings underline the multifactorial pathogenesis of rHCC. The observed incidence of $\mathrm{rHCC}$ is $5.5 \%$. Despite the inherent limitations, the risk score could help in guiding prophylactic treatment of high-risk HCC cases and aid in the triage of patients so that high-risk patients are given priority.

\section{ACKNOWLEDGEMENTS}

We thank Drs. Ying-Qiang Liu and Xu-Xin Yan from Department of Radiology in Ruijin Hospital for their cooperation in data collection.

\section{Fund/Grant Support}

This work was supported by the Scientific Research Fund Projects of Shanghai Science and Technology Committee (grant number 18441901800).

\section{Conflict of Interest}

No potential conflict of interest relevant to this article was reported.

\section{ORCID iD}

Feng Ye: https://orcid.org/0000-0002-2127-4604

Di Ma: https://orcid.org/0000-0002-2937-1839

Xiao-Yong Gong: https://orcid.org/0000-0002-0346-5677

Yu-Chen Yang: https://orcid.org/0000-0002-5962-0437

Yong-Jun Chen: https://orcid.org/0000-0002-6486-2000

\section{Author Contribution \\ Conceptualization: FY, DM, YJC \\ Formal Analysis: FY, XYG \\ Investigation: FY, YCY \\ Methodology: FY, YCY \\ Project Administration: FY, DM, YJC \\ Writing - Original Draft: FY, XYG, YCY \\ Writing - Review \& Editing: FY, DM, YJC}

\section{REFERENCES}

1. Zhu Q, Qiao G, Xu C, Yu X, Zhao J, Yu Z, et al. Conditional survival in patients with spontaneous tumor rupture of hepatocellular carcinoma after partial hepatectomy: a propensity score matching analysis. HPB (Oxford) 2019;21:722-30.

2. Sahu SK, Chawla YK, Dhiman RK, Singh V, Duseja A, Taneja S, et al. Rupture of hepatocellular carcinoma: a review of literature. J Clin Exp Hepatol 2019;9:24556.

3. Jeng KS, Huang CC, Lin CC, Lin CK, Teng $\mathrm{CJ}$, Chen $\mathrm{KH}$. Liver transplantation after downstagings of ruptured advanced hepatocellular carcinoma in cirrhotic liver: is it advisable? A case report. Transplant Proc 2019:51:1468-71.

4. Moris D, Chakedis J, Sun SH, Spolverato G, Tsilimigras DI, Ntanasis-Stathopoulos I, et al. Management, outcomes, and prognostic factors of ruptured hepatocellular carcinoma: a systematic review. J Surg Oncol 2018;117:341-53

5. Pillai A, Couri T, Charlton M. Liver allocation policies in the USA: past, present, and the future. Dig Dis Sci 2019;64:985-92.

6. Kerdsuknirun J, Vilaichone V, Vilaichone RK. Risk factors and prognosis of spontaneously ruptured hepatocellular carcinoma in Thailand. Asian Pac J Cancer Prev 2018;19:3629-34.

7. Li J, Huang L, Liu CF, Cao J, Yan JJ, Xu F, et al. Risk factors and surgical outcomes for spontaneous rupture of BCLC stages A and B hepatocellular carcinoma: a casecontrol study. World J Gastroenterol 2014:20:9121-7.

8. Cook NR. Use and misuse of the receiver operating characteristic curve in risk prediction. Circulation 2007;115:928-35.

9. Justice AC, Covinsky KE, Berlin JA. Assessing the generalizability of prognostic information. Ann Intern Med 1999;130:515-24.

10. Daskivich T, Luu M, Noah B, Fuller G, Anger J, Spiegel B. Differences in online consumer ratings of health care providers across medical, surgical, and allied health specialties: observational study of 212,933 providers. J Med Internet Res 2018;20:e176.

11. Kang L, Liu A, Tian L. Linear combination methods to improve diagnostic/prognostic accuracy on future observations. Stat Methods Med Res 2016;25:1359-80.

12. Chua DW, Koh YX, Allen JC, Chan CY, Lee SY, Cheow PC, et al. Impact of spontaneous rupture on the survival outcomes after liver resection for 
hepatocellular carcinoma: a propensity matched analysis comparing ruptured versus non-ruptured tumors. Eur J Surg Oncol 2019:45:1652-9.

13. Zheng J, Chou JF, Gönen M, Vachharajani N, Chapman WC, Majella Doyle MB, et al. Prediction of hepatocellular carcinoma recurrence beyond Milan criteria after resection: validation of a clinical risk score in an international cohort. Ann Surg 2017;266:693-701.

14. Ren A, Luo S, Ji L, Yi X, Liang J, Wang J, et al. Peritoneal metastasis after emergency hepatectomy and delayed hepatectomy for spontaneous rupture of hepatocellular carcinoma. Asian J Surg 2019;42:464-69.

15. Zhu Q, Li J, Yan JJ, Huang L, Wu MC, Yan YQ. Predictors and clinical outcomes for spontaneous rupture of hepatocellular carcinoma. World J Gastroenterol 2012;18:7302-7.

16. Aoki T, Kokudo N, Matsuyama Y, Izumi N, Ichida T, Kudo M, et al. Prognostic impact of spontaneous tumor rupture in patients with hepatocellular carcinoma: an analysis of 1160 cases from a nationwide survey. Ann Surg 2014:259:532-42.

17. Liu C, Xiao GQ, Yan LN, Li B, Jiang L, Wen TF, et al. Value of $\alpha$-fetoprotein in association with clinicopathological features of hepatocellular carcinoma. World J Gastroenterol 2013;19:1811-9.

18. Zhu L, Geng X, Fan S. Vascular elasticity in patients with spontaneous rupture of hepatocellular carcinoma. Zhonghua Gan Zang Bing Za Zhi 2002;10:96.

19. Wu J, Tong RL, Ding CF, Zhou J, Xu XF, Wang WL, et al. Establishment of prediction model for spontaneous rupture of primary liver cancer. Zhejiang Da Xue Xue Bao Yi Xue Ban 2014:43:652-7.

20. Chernyak V, Santillan CS, Papadatos D, Sirlin CB. LI-RADS ${ }^{\circledR}$ algorithm: CT and MRI. Abdom Radiol (NY) 2018:43:111-26. 